\title{
Deterministic and Stochastic Fractional-Order Hastings-Powell Food Chain Model
}

\author{
Moustafa El-Shahed ${ }^{1, *}$ and Asmaa M. Al-Dububan ${ }^{2}$
}

\author{
${ }^{1}$ Department of Mathematics, Unaizah College of Sciences and Arts, Qassim University, Unaizah, 51911, Saudi Arabia \\ ${ }^{2}$ Department of Mathematics, Buraydah College of Sciences and Arts, Qassim University, Buraydah, 51431, Saudi Arabia \\ ${ }^{*}$ Corresponding Author: Moustafa El-Shahed. Email: elshahedm@yahoo.com \\ Received: 09 April 2021; Accepted: 11 June 2021
}

\begin{abstract}
In this paper, a deterministic and stochastic fractional-order model of the tri-trophic food chain model incorporating harvesting is proposed and analysed. The interaction between prey, middle predator and top predator population is investigated. In order to clarify the characteristics of the proposed model, the analysis of existence, uniqueness, non-negativity and boundedness of the solutions of the proposed model are examined. Some sufficient conditions that ensure the local and global stability of equilibrium points are obtained. By using stability analysis of the fractional-order system, it is proved that if the basic reproduction number $R_{0}<1$, the predator free equilibrium point $E_{1}$ is globally asymptotically stable. The occurrence of local bifurcation near the equilibrium points is investigated with the help of Sotomayor's theorem. Some numerical examples are given to illustrate the theoretical findings. The impact of harvesting on prey and the middle predator is studied. We conclude that harvesting parameters can control the dynamics of the middle predator. A numerical approximation method is developed for the proposed stochastic fractional-order model.
\end{abstract}

Keywords: Food chain model; global dynamics; stability; stochastic; Hastings-Powell model; harvesting

\section{Introduction}

Mathematical analysis is one of the important tools for understanding and interpreting different interactions in the environment around us. The food chain model system is attractive to researchers in theoretical ecology because it helps to understand the relationships between populations and describe the behavior of the ecosystem. Hastings et al. [1] investigated a threespecies food chain model with Holling type II functional responses. Hastings-Powell model, has been revisited recently by many authors [2-16]. In the real world, food chain models are always affected by environmental noise. Thus, the stochastic models may be a more appropriate way of modeling the Hastings-Powell food chain model in many circumstances [17,18]. Recently, fractional calculus has been applied to describe different mathematical models, and it has been shown to be more accurate in some cases compared to the classical models [19-26]. The main objective

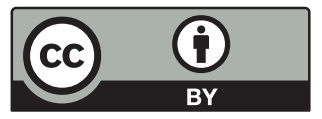

This work is licensed under a Creative Commons Attribution 4.0 International License, which permits unrestricted use, distribution, and reproduction in any medium, provided the original work is properly cited. 
of this paper is to study the deterministic and stochastic fractional-order Hastings-Powell model incorporating harvesting. This model is an extension of the classical Hastings-Powell model (1) by introducing fractional derivative, stochastic and harvesting. The qualitative behavior of the proposed model is investigated. Also, a numerical approximation method is developed for the proposed stochastic fractional-order model.

The paper is arranged as follows: In Section 2, the mathematical model is described. Some preliminary results, such as existence, uniqueness, nonnegativity and boundedness are presented in Section 3. The local and global stability of equilibrium points of the fractional-order food chain model is analyzed in Section 4. With the help of Sotomayor's theorem, the transcritical bifurcation of the proposed model is investigated in Section 5. Section 6 extends the deterministic fractionalorder food chain model to the stochastic fractional-order model. In Section 7, some numerical simulations are presented to verify the obtained theoretical results. Finally, the conclusions are given in Section 8.

\section{Mathematical Model}

Recently, Nath et al. [27] studied the following system of integer order differential equations to understand the underlying dynamics of the food chain model:

$\frac{d X}{d T}=r X\left(1-\frac{X}{k}\right)-\frac{\beta X Y}{A+X}-H_{1} X$,

$\frac{d Y}{d T}=\frac{e_{1} \beta X Y}{A+X}-\frac{\gamma Y Z}{B+Y}-d Y-H_{2} Y^{2}$,

$\frac{d Z}{d T}=\frac{e_{2} \gamma Y Z}{B+Y}-\delta Z-H_{3} Z$,

with initial values $X(0)=X_{0} \geq 0, Y(0)=Y_{0} \geq 0, Z(0)=Z_{0} \geq 0$.

In the above model $X(T), Y(T)$ and $Z(T)$ represent the population density of prey, middle predator and top predator at time $T$, respectively. The prey grows with an intrinsic growth rate $r$ and carrying capacity $k$ in the absence of predation. It is assumed that the middle predator consumes prey following Holling type II functional response, and $\beta$ is the maximum predation rate of the middle predator in prey. The middle predator is attacked by the top predator at rate $\gamma$ using Holling type II functional response. $A$ and $B$ are the half saturation constants. $e_{1}$ and $e_{2}$ are conversion rate for middle predator and top predator, respectively. $d$ and $\delta$ are the mortality rate of the middle predator and top predator, respectively. Here $H_{1}$ and $H_{3}$ are the harvesting rate of the prey population and top predator, respectively. The term $H_{2} Y^{2}$ represents the quadratic harvesting of the middle predator.

Introducing dimensionless

$x=\frac{X}{k}, \quad y=\frac{Y}{k e_{1}}, \quad z=\frac{Z}{k e_{1} e_{2}}, \quad t=r T$.

Making the above substitutions in the model (1). Then the system yields the following form $\frac{d x}{d t}=x(1-x)-\frac{a_{1} x y}{1+b_{1} x}-h x$, 
$\frac{d y}{d t}=\frac{a_{1} x y}{1+b_{1} x}-\frac{a_{2} y z}{1+b_{2} y}-\mu_{1} y-\eta y^{2}, \quad A=\pi r^{2}$

$\frac{d z}{d t}=\frac{a_{2} y z}{1+b_{2} y}-\mu_{2} z$.

The dimensionless parameters in the food chain model (2) are defined as

$a_{1}=\frac{k \beta e_{1}}{r A}, \quad a_{2}=\frac{k \gamma e_{1} e_{2}}{r B}, \quad b_{1}=\frac{k}{A}, \quad b_{2}=\frac{k e_{1}}{B}, \quad h=\frac{H_{1}}{r}, \quad \mu_{1}=\frac{d}{r}, \quad \mu_{2}=\frac{\delta+H_{3}}{r}$,

$\eta=\frac{k e_{1} H_{2}}{r}$

Following $[13,15,16]$, by replacing the integer derivative by a Caputo fractional derivative in (2), one can obtain the following fractional-order system

${ }^{c} D^{\alpha} x=x(\gamma-x)-\frac{a_{1} x y}{1+b_{1} x}$,

${ }^{c} D^{\alpha} y=\frac{a_{1} x y}{1+b_{1} x}-\frac{a_{2} y z}{1+b_{2} y}-\mu_{1} y-\eta y^{2}$,

${ }^{c} D^{\alpha} z=\frac{a_{2} y z}{1+b_{2} y}-\mu_{2} z$

where, $0<\alpha<1$ and $\gamma=1-h$.

\section{Some Preliminary Results}

\subsection{Existence and Uniqueness}

In this section, the existence and uniqueness of the solutions of the fractional-order system (3) are investigate in the region $\Omega \times(0, T]$ where

$\Omega=\left\{(x, y, z) \in \mathbb{R}_{+}^{3}: \max (|x|,|y|,|z|) \leq \varphi\right\}$,

for sufficiently large $\varphi$.

Theorem 1. For each $X_{0}=\left(x_{0}, y_{0}, z_{0}\right) \in \Omega$, there exists a unique solution $X(t) \in \Omega$ of the fractional-order system (3), which is defined for all $t \geq 0$.

Proof. Define a mapping $F(X)=\left(F_{1}(X), F_{2}(X), F_{3}(X)\right)$, in which

$F_{1}(X)=x(\gamma-x)-\frac{a_{1} x y}{1+b_{1} x}$,

$F_{2}(X)=\frac{a_{1} x y}{1+b_{1} x}-\frac{a_{2} y z}{1+b_{2} y}-\mu_{1} y-\eta y^{2}$,

$F_{3}(X)=\frac{a_{2} y z}{1+b_{2} y}-\mu_{2} z$. 
For any $X, \bar{X} \in \Omega$, it follows from (3) that

$$
\begin{aligned}
\|F(X)-F(\bar{X})\|= & \left|F_{1}(X)-F_{1}(\bar{X})\right|+\left|F_{2}(X)-F_{2}(\bar{X})\right|+\left|F_{3}(X)-F_{3}(\bar{X})\right| \\
= & \left|x(\gamma-x)-\frac{a_{1} x y}{1+b_{1} x}-\bar{x}(\gamma-\bar{x})+\frac{a_{1} \overline{x y}}{1+b_{1} \bar{x}}\right| \\
& +\left|\frac{a_{1} x y}{1+b_{1} x}-\frac{a_{2} y z}{1+b_{2} y}-\mu_{1} y-\eta y^{2}-\frac{a_{1} \overline{x y}}{1+b_{1} \bar{x}}+\frac{a_{2} \overline{y z}}{1+b_{2} \bar{y}}+\mu_{1} \bar{y}+\eta \bar{y}^{2}\right| \\
& +\left|\frac{a_{2} y z}{1+b_{2} y}-\mu_{2} z-\frac{a_{2} \overline{y z}}{1+b_{2} \bar{y}}+\mu_{2} \bar{z}\right| \\
\leq & \left(\gamma+2 \varphi+2 a_{1} \varphi\right)|x-\bar{x}|+\left(\mu_{1}+2 \eta \varphi+2 a_{1} \varphi+\frac{2 a_{1}}{b_{1}}+2 a_{2} \varphi\right)|y-\bar{y}| \\
& +\left(\mu_{2}+\frac{4 a_{2}}{b_{2}}\right)|z-\bar{z}| \\
\leq & M_{1}\|X-\bar{X}\|,
\end{aligned}
$$

where

$M_{1}=\max \left\{\gamma+2 \varphi\left(1+a_{1}\right), \mu_{1}+2 \eta \varphi+2 a_{1} \varphi+\frac{2 a_{1}}{b_{1}}+2 a_{2} \varphi, \mu_{2}+\frac{4 a_{2}}{b_{2}}\right\}$.

Hence, $F(X)$ satisfies the Lipschitz condition with respect to $X$. According to Cresson et al. [28], as $F(X)$ locally Lipschitz. Then there exists a unique local solution to the fractionalorder system (3).

\subsection{Non-Negativity and Boundedness}

The following results show the non-negativity of the solutions of the fractional-order system (3). According to [28], a model of the form $\frac{d X}{d t}=F(X)$ satisfies the positivity property if and only if for all $i=1,2,3, F_{i}(X) \geq 0$ for all $X \in R_{+}^{3}$ such that $X_{i}=0$. Thus, the solution of the integer-order model (1), with nonnegative initial conditions remains nonnegative. Also, the solution satisfies the Lipschitz condition, as stated in Theorem 1. By Theorem 5 and Theorem 6 in Cresson et al. [28], the solution of the fractional-order model (3) also satisfies the non-negativity. The boundedness of the solutions of model (3) are given in the following theorem.

Theorem 2. All the solutions of the fractional-order food chain Model (3) starting in $\mathbb{R}_{+}^{3}$ are uniformly bounded.

Proof. The approach of $[29,30]$ is utilized. Let $(x(t), y(t), z(t))$ to be any solution of the system (3) with non-negative initial conditions. Let $w(t)=x(t)+y(t)+z(t)$, then

$$
\begin{aligned}
{ }^{c} D^{\alpha} w(t) & \leq-x^{2}+\gamma x-\mu_{1} y-\mu_{2} z \\
& \leq-x^{2}+2 \gamma x-v(x+y+z),
\end{aligned}
$$


where, $v<\min \left\{\gamma, \mu_{1}, \mu_{2}\right\}$, thus, ${ }^{c} D^{\alpha} w(t)+v w \leq \gamma^{2}$. In accordance with Lemma 9 in Choi et al. [31], it follows that

$0 \leq w(t) \leq w(0) E_{\alpha}\left(-v t^{\alpha}\right)+\gamma^{2} t^{\alpha} E_{\alpha, \alpha+1}\left(-v t^{\alpha}\right)$,

where $E_{\alpha}$ is the Mittag-Leffler function. According to Lemma 5 and Corollary 6 in Choi et al. [31], it follows

$0 \leq w(t) \leq \frac{\gamma^{2}}{v}, \quad$ as $t \rightarrow \infty$

Hence all the solutions of fractional-order a tri-trophic food chain model (3) that start in $\mathbb{R}_{+}^{3}$ are uniformly bounded in the region

$H=\left\{(x, y, z) \in \mathbb{R}_{+}^{3}: w(t) \leq \frac{\gamma^{2}}{v}+\xi, \quad\right.$ for any $\left.\xi>0\right\}$.

One can also prove that $x \leq \gamma$.

\section{Equilibria and Stability}

The fractional-order system (3) has the following equilibrium points:

1) $E_{0}=(0,0,0)$, that is, the extinction of prey, middle predator, and top predator. The trivial equilibrium $E_{0}$ always exists.

2) The middle predator and top predator free equilibrium point $E_{1}=(\gamma, 0,0)$. Therefore $E_{1}$ exists if $h<1$. Using the next generation method, one can obtain the basic reproduction number $R_{0}=\frac{a_{1} \gamma}{\mu_{1}\left(1+b_{1} \gamma\right)}$.

3) Following [32], $R_{0}$ determine the local and global stability of $E_{1}$. Here $\frac{\gamma a_{1}}{\left(1+\gamma b_{1}\right)}$ and $\frac{1}{\mu_{1}}$ are the birth rate and the mean lifespan of middle predator at $E_{1}$, respectively. Subsequently their product gives the basic reproduction number at $E_{1}$. In the following, two critical parameters $R_{1}$ and $R_{2}$, can be used to classify the dynamics of the fractional-order model (3). The threshold parameter $R_{1}$ defined by $R_{1}=\frac{a_{1} x_{2}}{\mu_{1}\left(1+b_{1} x_{2}\right)}$, while the threshold parameter $R_{2}$ defined by $R_{2}=$ $\frac{a_{2} y_{2}}{\mu_{2}\left(1+b_{2} y_{2}\right)}$.

4) The top predator extinction equilibrium point $E_{2}=\left(x_{2}, y_{2}, 0\right)$, where $y_{2}=\frac{\mu_{1}}{\eta}\left(R_{1}-1\right)$, and $x_{2}$ satisfies the equation

$b_{1} x_{2}^{2}+\left(1-\gamma b_{1}\right) x_{2}+\frac{a_{1} \mu_{1}}{\eta}\left(R_{1}-1\right)-\gamma=0$. 
5) The top predator extinction equilibrium point $E_{2}$ exists if $b_{1} \gamma>1$ and $1+\frac{\gamma \eta}{a_{1} \mu_{1}}<R_{1}<$ $1+\frac{\eta\left(1+b_{1} \gamma\right)^{2}}{4 a_{1} b_{1} \mu_{1}}$

6) The coexistence positive equilibrium point $E_{3}=\left(x_{3}, y_{3}, z_{3}\right)$, where

$y_{3}=\frac{\mu_{2}}{a_{2}-\mu_{2} b_{2}}, \quad z_{3}=\frac{\left(1+b_{2} y_{3}\right)}{a_{2}}\left(\frac{a_{1} x_{3}}{1+b_{1} x_{3}}-\mu_{1}-\eta y_{3}\right)$,

7) and $x_{3}$ is the positive root of the equation:

$b_{1} x_{3}^{2}+\left(1-\gamma b_{1}\right) x_{3}+\left(a_{1} y_{3}-\gamma\right)=0$.

$E_{3}\left(x_{3}, y_{3}, z_{3}\right)$ exists if

$a_{2}>\mu_{2} b_{2}, \quad \frac{a_{1} x_{3}}{1+b_{1} x_{3}}>\mu_{1}+\eta y_{3}, \quad b_{1} \gamma>1, \quad a_{1} y_{3}>\gamma, \quad\left(1-\gamma b_{1}\right)^{2}>4 b_{1}\left(a_{1} y_{3}-\gamma\right)$.

The locally and globally asymptotically stable of equilibrium points of fractional-order food chain model (3) are now investigated. The Jacobian matrix is given as follows:

$$
J(x, y, z)=\left(\begin{array}{lll}
\gamma-2 x-\frac{a_{1} y}{\left(1+b_{1} x\right)^{2}} & \frac{-a_{1} x}{1+b_{1} x} & 0 \\
\frac{a_{1} y}{\left(1+b_{1} x\right)^{2}} & \frac{a_{1} x}{1+b_{1} x}-\frac{a_{2} z}{\left(1+b_{2} y\right)^{2}}-\mu_{1}-2 \eta y & \frac{-a_{2} y}{1+b_{2} y} \\
0 & \frac{a_{2} z}{\left(1+b_{2} y\right)^{2}} & \frac{a_{2} y}{1+b_{2} y}-\mu_{2}
\end{array}\right) .
$$

The eigenvalues of $J$ around $E_{0}$ are $\gamma,-\mu_{1}$ and $-\mu_{2}$, therefore $E_{0}$ is unstable saddle point. The stability of middle predator extinction equilibrium point $E_{1}$ is investigated as follows

Theorem 3. If $R_{0}<1$, then $E_{1}$ is locally asymptotically stable.

Proof. The Jacobian matrix of model (3) at $E_{1}$ is

$$
J\left(E_{1}\right)=\left(\begin{array}{lll}
-\gamma & \frac{-a_{1} \gamma}{1+b_{1} \gamma} & 0 \\
0 & \frac{a_{1} \gamma}{1+b_{1} \gamma}-\mu_{1} & 0 \\
0 & 0 & -\mu_{2}
\end{array}\right) .
$$

The eigenvalues of $J\left(E_{1}\right)$ are $-\gamma,-\mu_{2}$ and $\frac{a_{1} \gamma}{1+b_{1} \gamma}-\mu_{1}$. Thus, $E_{1}$ is locally asymptotically stable if $\frac{a_{1} \gamma}{1+b_{1} \gamma}<\mu_{1}$, which is equivalent to $R_{0}<1$.

Theorem 4. If $R_{0}<1$, then $E_{1}$ is globally asymptotically stable. 
Proof. Let us consider the following positive definite Lyapunov function $V_{1}=\frac{L_{1}}{2}(x-\gamma)^{2}+\frac{1}{2} y^{2}+y+z$.

The time derivative of $V_{1}$ along the solution of fractional-order tri-trophic food chain model (3), one obtains

$$
\begin{aligned}
{ }^{c} D^{\alpha} V_{1} \leq & L_{1}(x-\gamma) \frac{d x}{d t}+(1+y) \frac{d y}{d t}+\frac{d z}{d t} \\
\leq & L_{1}(x-\gamma)\left(x(\gamma-x)-\frac{a_{1} x y}{1+b_{1} x}\right)+(1+y)\left[\frac{a_{1} x y}{1+b_{1} x}-\frac{a_{2} y z}{1+b_{2} y}-\mu_{1} y-\eta y^{2}\right] \\
& +\left(\frac{a_{2} y z}{1+b_{2} y}-\mu_{2} z\right) \\
\leq & -L_{1} x(x-\gamma)^{2}+\left(\frac{a_{1} x}{1+b_{1} x}-\mu_{1}\right) y^{2}+\left(\frac{L_{1} a_{1} \gamma x}{1+b_{1} x}+\frac{a_{1} x}{1+b_{1} x}-\mu_{1}\right) y .
\end{aligned}
$$

According to Theorem $1, x \leq \gamma$, and consequently $\frac{a_{1} x}{1+b_{1} x} \leq \frac{a_{1} \gamma}{1+b_{1} \gamma}$. The above inequality can be written as

${ }^{c} D^{\alpha} V_{1} \leq\left(\frac{a_{1} \gamma}{1+b_{1} \gamma}-\mu_{1}\right) y^{2}+\left(\frac{L_{1} a_{1} \gamma^{2}}{1+b_{1} \gamma}+\frac{a_{1} \gamma}{1+b_{1} \gamma}-\mu_{1}\right) y$.

Choosing $L_{1}=\frac{1-R_{0}}{\gamma R_{0}}$, one obtains ${ }^{c} D^{\alpha} V_{1} \leq 0$.

According to generalized Lyapunov-Lasalle's invariance principle [33], $E_{1}$ is globally asymptotically stable.

Hence the equilibrium point $E_{1}$ is globally asymptotically stable if $R_{0}<1$.

The global stability of the equilibrium point $E_{1}$ can be further demonstrated from the second equation of the system (3) as follows

${ }^{c} D^{\alpha} y \leq\left(\frac{a_{1} x}{1+b_{1} x}-\mu_{1}\right) y \leq\left(\frac{a_{1} \gamma}{1+b_{1} \gamma}-\mu_{1}\right) y \leq \mu_{1}\left(R_{0}-1\right) y$,

when $R_{0}<1, y(t) \rightarrow \infty$. The extinction of middle predator $y(t)$ implies the extinction of top predator $z(t)$. Furthermore, the equilibrium point $E_{1}$ is globally asymptotically stable. Following [32], $R_{0}<1$, implies that the conversion of prey into middle predator during lifespan of middle predator is less than unity. As a result, the biomass flow from prey to middle predator and top predator will stop. In this case, the middle predator and top predator will be extinct.

The stability of top predator extinction equilibrium point $E_{2}$ is investigated as follows

Theorem 5. If $R_{2}<1$ and $\frac{a_{1} b_{1} y_{2}}{\left(1+b_{1} x_{2}\right)^{2}}<1$ then the top predator extinction equilibrium point $E_{2}$ is locally asymptotically stable. 
Proof. The Jacobian matrix of model (3) at $E_{2}$ is

$$
J\left(E_{2}\right)=\left(\begin{array}{lll}
\Upsilon & \frac{-a_{1} x_{2}}{1+b_{1} x_{2}} & 0 \\
\frac{a_{1} y_{2}}{\left(1+b_{1} x_{2}\right)^{2}} & -\eta y_{2} & \frac{-a_{2} y_{2}}{1+b_{2} y_{2}} \\
0 & 0 & \frac{a_{2} y_{2}}{1+b_{2} y_{2}}-\mu_{2}
\end{array}\right),
$$

where $\Upsilon=\gamma-2 x_{2}-\frac{a_{1} y_{2}}{\left(1+b_{1} x_{2}\right)^{2}}$. The eigenvalues of $J\left(E_{2}\right)$ are the roots of equation

$$
\left(\frac{a_{2} y_{2}}{1+b_{2} y_{2}}-\mu_{2}-\lambda\right)\left(\lambda^{2}+\left(\eta y_{2}-\Upsilon\right) \lambda+\frac{a_{1}^{2} x_{2} y_{2}}{\left(1+b_{1} x_{2}\right)^{3}}-\eta y_{2} \Upsilon\right)=0
$$

The above equation has the following eigenvalue $\lambda_{1}=\frac{a_{2} y_{2}}{1+b_{2} y_{2}}-\mu_{2}=\mu_{2}\left(R_{2}-1\right)$. The eigenvalues $\lambda_{2,3}$ have negative real parts if $\Upsilon<0$. Thus, if $R_{2}<1$ and $\Upsilon<0$, then the top predator extinction equilibrium point $E_{2}$ is locally asymptotically stable.

Theorem 6. If $\frac{a_{1} b_{1} y_{2}}{\left(1+b_{1} x_{2}\right)}<1$ and $a_{2} y_{2}<\mu_{2}$, then the top predator extinction equilibrium point $E_{2}$ is globally asymptotically stable.

Proof. The following positive definite Lyapunov function is considered. $V_{2}=L_{2}\left(x-x_{2}-x_{2} \ln \frac{x}{x_{2}}\right)+y-y_{2}-y_{2} \ln \frac{y}{y_{2}}+z$.

By calculating the time derivative of $V_{2}$ along the solution of system (3), one obtains,

$$
\begin{aligned}
{ }^{c} D^{\alpha} V_{2} \leq & L_{2}\left(x-x_{2}\right)\left[\gamma-x-\frac{a_{1} y}{1+b_{1} x}\right]+\left(y-y_{2}\right)\left[\frac{a_{1} x}{1+b_{1} x}-\frac{a_{2} z}{1+b_{2} y}-\mu_{1}-\eta y\right]+z\left(\frac{a_{2} y}{1+b_{2} y}-\mu_{2}\right) \\
\leq & L_{2}\left(x-x_{2}\right)^{2}\left(\frac{a_{1} b_{1} y_{2}}{\left(1+b_{1} x\right)\left(1+b_{1} x_{2}\right)}-1\right)+\frac{\left[a_{1}-a_{1} L_{2}-a_{1} b_{1} L_{2} x_{2}\right]}{\left(1+b_{1} x\right)\left(1+b_{1} x_{1}\right)}\left(x-x_{2}\right)\left(y-y_{2}\right) \\
& +z\left(a_{2} y_{2}-\mu_{2}\right) .
\end{aligned}
$$

Choosing $L_{2}=\frac{1}{1+b_{1} x_{2}}$, then ${ }^{c} D^{\alpha} V_{2} \leq 0$ when $\frac{a_{1} b_{1} y_{2}}{\left(1+b_{1} x_{2}\right)}<1$ and $a_{2} y_{2}<\mu_{2}$, and hence, according to generalized Lyapunov-Lasalle's invariance principle [33], the top predator extinction equilibrium point $E_{2}$ is globally asymptotically stable when $\frac{a_{1} b_{1} y_{2}}{\left(1+b_{1} x_{2}\right)}<1$ and $a_{2} y_{2}<\mu_{2}$.

The stability of coexistence equilibrium point $E_{3}$ is investigated as follows.

Theorem 7. If $\frac{a_{1} b_{1} y_{3}}{\left(1+b_{1} x_{3}\right)^{2}}<1$ and $\frac{a_{2} b_{2} z_{3}}{\left(1+b_{2} y_{3}\right)^{2}}<\eta$, then the coexistence equilibrium point $E_{3}$ is locally asymptotically stable. 
Proof. The Jacobian matrix of model (3) at $E_{3}$ is

$J\left(E_{3}\right)=\left(\begin{array}{lll}C_{1} & \frac{-a_{1} x_{3}}{1+b_{1} x_{3}} & 0 \\ \frac{a_{1} y_{3}}{\left(1+b_{1} x_{3}\right)^{2}} & C_{2} & \frac{-a_{2} y_{3}}{1+b_{2} y_{3}} \\ 0 & \frac{a_{2} z_{3}}{\left(1+b_{2} y_{3}\right)^{2}} & 0\end{array}\right)$,

where $C_{1}=\frac{a_{1} b_{1} x_{3} y_{3}}{\left(1+b_{1} x_{3}\right)^{2}}-x_{3}$ and $C_{2}=\frac{a_{2} b_{2} y_{3} z_{3}}{\left(1+b_{2} y_{3}\right)^{2}}-\eta y_{3}$. The characteristic polynomial of $J\left(E_{3}\right)$ is

$\lambda^{3}+\phi_{1} \lambda^{2}+\phi_{2} \lambda+\phi_{3}=0$,

where

$\phi_{1}=-\left(C_{1}+C_{2}\right)$,

$\phi_{2}=\left[\frac{a_{1}^{2} x_{3} y_{3}}{\left(1+b_{1} x_{3}\right)^{3}}+\frac{a_{2}^{2} y_{3} z_{3}}{\left(1+b_{2} y_{3}\right)^{3}}+C_{1} C_{2}\right]$,

$\phi_{3}=\frac{-a_{2}^{2} C_{1} y_{3} z_{3}}{\left(1+b_{2} y_{3}\right)^{3}}$.

If we choose $C_{1}<0$ and $C_{2}<0$, then $\phi_{1}>0, \phi_{2}>0, \phi_{3}>0$ and $\phi_{1} \phi_{2}>\phi_{3}$. According to Routh-Hurtwitz criteria, the system (3) is locally asymptotically stable around the coexistence equilibrium point $E_{3}$, when $\frac{a_{1} b_{1} y_{3}}{\left(1+b_{1} x_{3}\right)^{2}}<1$ and $\frac{a_{2} b_{2} z_{3}}{\left(1+b_{2} y_{3}\right)^{2}}<\eta$.

Following [34], it is interested to note that the function

$\Phi_{i}(\alpha, \theta)=\frac{\alpha \pi}{2}-\left|\arg \left(\lambda_{i}(\theta)\right)\right|, \quad i=1,2,3$,

has a similar effect as the real part of the eigenvalue in the integer order system. If $\Phi_{i}(\alpha, \theta)<0$ for all $i=1,2,3$, then $E_{3}$ is locally asymptotically stable. If there exist $i$ such that $\Phi_{i}(\alpha, \theta)>0$, then $E_{3}$ is unstable.

Theorem 8. If $\frac{a_{1} b_{1} y_{3}}{1+b_{1} x_{3}}<1$ and $\frac{a_{2} b_{2} z_{3}}{1+b_{1} y_{3}}<\eta$, then the coexistence equilibrium point $E_{3}$ is globally asymptotically stable.

Proof. Let us consider the function

$V_{3}=L_{3}\left(x-x_{3}-x_{3} \ln \frac{x}{x_{3}}\right)+\left(y-y_{3}-y_{3} \ln \frac{y}{y_{3}}\right)+L_{4}\left(z-z_{3}-z_{3} \ln \frac{z}{z_{3}}\right)$. 
By calculating the time derivative of $V_{3}$ along along with the solution of system (3), one obtains,

$$
\begin{aligned}
{ }^{c} D^{\alpha} V_{3} \leq & L_{3}\left(x-x_{3}\right)\left[\gamma-x-\frac{a_{1} y}{1+b_{1} x}\right]+\left(y-y_{3}\right)\left[\frac{a_{1} x}{1+b_{1} x}-\frac{a_{2} z}{1+b_{2} y}-\mu_{1}-\eta y\right] \\
& +L_{4}\left(z-z_{3}\right)\left[\frac{a_{2} y}{1+b_{2} y}-\mu_{2}\right] \\
= & L_{3}\left(x-x_{3}\right)\left[\left(x_{3}-x\right)+\frac{a_{1} y_{3}}{1+b_{1} x_{3}}-\frac{a_{1} y}{1+b_{1} x}\right]+L_{4}\left(z-z_{3}\right)\left[\frac{a_{2} y}{1+b_{2} y}-\frac{a_{2} y_{3}}{1+b_{2} y_{3}}\right] \\
& +\left(y-y_{3}\right)\left[\frac{a_{1} x}{1+b_{1} x}-\frac{a_{1} x_{3}}{1+b_{1} x_{3}}+\frac{a_{2} z_{3}}{1+b_{2} y_{3}}-\frac{a_{2} z}{1+b_{2} y}+\eta\left(y_{3}-y\right)\right] \\
= & L_{3}\left(\frac{a_{1} b_{1} y_{3}}{\left(1+b_{1} x\right)\left(1+b_{1} x_{3}\right)}-1\right)\left(x-x_{3}\right)^{2}+\frac{\left(x-x_{3}\right)\left(y-y_{3}\right)}{\left(1+b_{1} x\right)\left(1+b_{1} x_{3}\right)}\left[a_{1}-a_{1} L_{3}-a_{1} b_{1} L_{3} x_{3}\right] \\
& +\frac{\left(y-y_{3}\right)\left(z-z_{3}\right)}{\left(1+b_{2} y\right)\left(1+b_{2} y_{3}\right)}\left[a_{2} L_{4}-a_{2}-a_{2} b_{2} y_{3}\right]+\left(\frac{a_{2} b_{2} z_{3}}{\left(1+b_{2} y\right)\left(1+b_{2} y_{3}\right)}-\eta\right)\left(y-y_{3}\right)^{2} .
\end{aligned}
$$

Choosing $L_{3}=\frac{1}{1+b_{1} x_{3}}$ and $L_{4}=1+b_{2} y_{3}$, then ${ }^{c} D^{\alpha} V_{3} \leq 0$ when $\frac{a_{1} b_{1} y_{3}}{1+b_{1} x_{3}}<1$ and $\frac{a_{2} b_{2} z_{3}}{1+b_{2} y_{3}}<$ $\eta$. Thus, according to generalized Lyapunov-Lasalle's invariance principle [33], the coexistence equilibrium point $E_{3}$ is globally asymptotically stable.

\section{Bifurcation Analysis}

In this section we will investigate the local bifurcation near the free predator equilibrium point of the food chain model (2) with the help of Sotomayor's theorem [35] to discuss the bifurcation analysis of the underlying system. The food chain model (2) can be rewritten in a vector form $\frac{d X}{d t}=F(X)$, where $X=(x, y, z)^{T}$ and $F=\left(F_{1}, F_{2}, F_{3}\right)^{T}$ with $F_{i}, i=1,2,3$ are given in (4).

Theorem 9. The food chain model (2) undergoes a transcritical bifurcation with respect to the bifurcation parameter $\mu_{1}$ around the free predator equilibrium point $E_{1}=(\gamma, 0,0)$ if $R_{0}=1$.

Proof. The Jacobian matrix of the food chain model (2) at the free predator equilibrium point $E_{1}$ with $\mu_{1}=\mu_{1}^{*}=\frac{\gamma a_{1}}{1+b_{1} \gamma}$ has zero eigenvalue takes the form

$J\left(E_{1}\right)=\left(\begin{array}{lll}-\gamma & -\frac{\gamma a_{1}}{1+b_{1} \gamma} & 0 \\ 0 & 0 & 0 \\ 0 & 0 & -\mu_{2}\end{array}\right)$.

The eigenvector corresponding to $J\left(E_{1}\right) W_{1}=0$ is $W_{1}=\left(v_{1},-\frac{\left(1+b_{1} \gamma\right)}{a_{1}}, 0\right)^{T}$ where $v_{1}$ is any non zero real number. Similarly, the eigenvector corresponding to $J\left(E_{1}\right)^{T} W_{2}=0$ is given by 
$W_{2}=\left(0, \tau_{2}, 0\right)^{T}$, where $\tau_{2}$ is any non zero real number. Consider, $\frac{\partial F}{\partial \mu_{1}}=F_{\mu_{1}}\left(X, \mu_{1}\right)=(0,-y, 0)^{T}$, thus, $W_{2}^{T} F_{\mu_{1}}\left(E_{1}, \mu_{1}^{*}\right)=0$. Therefore, according to Sotomayor's theorem for local bifurcation, the food chain model (2) has no saddle-node bifurcation near $E_{1}$ at $\mu_{1}^{*}=\frac{\gamma a_{1}}{1+b_{1} \gamma}$. Now,

$D F_{\mu_{1}}\left(E_{1}, \mu_{1}^{*}\right)=\left(\begin{array}{lll}0 & 0 & 0 \\ 0 & -1 & 0 \\ 0 & 0 & 0\end{array}\right)$

then $W_{2}^{T} D F_{\mu_{1}}\left(E_{1}, \mu_{1}^{*}\right) W_{1}=\frac{\nu_{1} \tau_{2}\left(b_{1} \gamma+1\right)}{a_{1}} \neq 0$, and $W_{2}^{T} D^{2} F\left(X, \mu_{1}\right)\left(W_{1}, W_{1}\right)=\tau_{2}\left(\frac{2 a_{1} v_{1} v_{2}}{\left(b_{1} \gamma+1\right)^{2}}-2 a_{2} v_{3} v_{2}-2 \eta v_{2}^{2}\right) \neq 0$.

Thus, according to Sotomayor's theorem, the food chain model (2) has a transcritical bifurcation at $\mu_{1}^{*}=\frac{\gamma a_{1}}{1+b_{1} \gamma}$ as the parameter $\mu_{1}$ passes through the value $\mu_{1}^{*}$, thus the proof is complete. It is interested to note that $R_{0}=1$ is equivalent to $\mu_{1}=\frac{\gamma a_{1}}{1+b_{1} \gamma}$.

\section{Stochastic Fractional-order Model}

This section extends the deterministic fractional-order food chain model (3) to the following stochastic fractional-order model.

$$
\begin{aligned}
& { }^{C} D^{\alpha} x=x(\gamma-x)-\frac{a_{1} x y}{1+b_{1} x}+\sigma_{1} x \frac{d \sigma_{1}}{d t}, \\
& { }^{C} D^{\alpha} y=\frac{a_{1} x y}{1+b_{1} x}-\frac{a_{2} y z}{1+b_{2} y}-\mu_{1} y-\eta y^{2}+\sigma_{2} y \frac{d \sigma_{2}}{d t}, \\
& { }^{C} D^{\alpha} z=\frac{a_{2} y z}{1+b_{2} y}-\mu_{2} z+\sigma_{3} z \frac{d \sigma_{3}}{d t},
\end{aligned}
$$

where $\sigma_{i}(i=1,2,3)$ are independent standard Brownian motions with $\sigma_{i}(0)=0$ and $\sigma_{i}>0$ denote the intensities of the white noise. The stochastic fractional-order food chain model (11) can be written in the general form:

${ }^{C} D^{\alpha} X(t)=F(X)+g(X) \frac{d \sigma}{d t}$,

where $F(X)$ is given in (4), $g(x)=\left(\sigma_{1} x, \sigma_{2} y, \sigma_{3} z\right)$ and $\frac{d \sigma}{d t}=\left(\frac{d \sigma_{1}}{d t}, \frac{d \sigma_{2}}{d t}, \frac{d \sigma_{3}}{d t}\right)^{T}$. Applying Riemann-Liouville integral to both sides of (12), one can obtain the following stochastic Volterra integral equation.

$$
X(t)=X_{0}+\int_{0}^{t} \frac{F(X)(t-s)^{\alpha-1}}{\Gamma(\alpha)} d s+\int_{0}^{t} \frac{g(X)(t-s)^{\alpha-1} d \sigma(s)}{\Gamma(\alpha)} d s
$$


According to [36-39], under some conditions on the coefficient functions, the global existence and uniqueness of solutions for the stochastic fractional-order system (13) can be investigated. Because Grunwald-Letnikov's definition is the most straightforward from the point of view of numerical implementation, so one can use it to solve the Hastings-Pwoell system of fractionalorder stochastic differential equations. Grunwald-Letnikov $\left({ }^{G L} D^{\alpha}\right)$ fractional derivative of order $\alpha$ defined by [40-43]

${ }^{G L} D^{\alpha} f(t)=\operatorname{Lim}_{h \rightarrow 0} h^{-\alpha} \sum_{j=0}^{\left[\frac{t-a}{h}\right]}(-1)^{j}\left(\begin{array}{l}\alpha \\ j\end{array}\right) f(t-j h)$,

where $\left[\frac{t-a}{h}\right]$ means the integer part of $\frac{t-a}{h}$. This formula can be reduced to

${ }^{G L} D^{\alpha} f\left(t_{n}\right) \approx h^{-\alpha} \sum_{j=0}^{n} w_{j}^{\alpha} f\left(t_{n-j}\right)$,

where $h$ is the time step, $t_{n}=n h$ and $w_{j}^{\alpha}$ are the Grunwald-Letnikov coefficients satisfy the following recurrence relationship

$w_{0}^{\alpha}=1, \quad w_{j}^{\alpha}=\left(1-\frac{1+\alpha}{j}\right) w_{j-1}^{\alpha}, \quad j=1,2,3, \ldots$

If $f(t)$ is continuous function and $f^{\prime}(t)$ is integrable function in the interval $[0, T]$, then the relation between Caputo and Grunwald-Letnikov fractional derivative takes the form [44-46]

$$
\begin{aligned}
{ }^{C} D^{\alpha} f(t) & ={ }^{G L} D^{\alpha} f(t)-\frac{f(0) t^{-\alpha}}{\Gamma(1-\alpha)} \\
& ={ }^{G L} D^{\alpha} f(t)-\operatorname{Lim}_{n \rightarrow \infty} \frac{(-1)^{n}}{h^{\alpha}}\left(\begin{array}{c}
\alpha-1 \\
n
\end{array}\right) f(0) \\
& \approx \frac{1}{h^{\alpha}} \sum_{j=0}^{n} w_{j}^{\alpha}\left(f\left(t_{n-j}\right)-f(0)\right) .
\end{aligned}
$$

Now, the fractional-order stochastic Hastings-Pwoell model (11) in Grunwald-Letnikov sense can be written as

$$
\begin{aligned}
& x_{n}=x_{0}+h^{\alpha}\left(x_{n-1}\left(\gamma-x_{n-1}\right)-\frac{a_{1} x_{n-1} y_{n-1}}{1+b_{1} x_{n-1}}+\sigma_{1} x_{n-1} \sqrt{h} \zeta_{1 n}\right)-\sum_{j=1}^{n} w_{j}^{\alpha}\left(x_{n-j}-x_{0}\right) \\
& y_{n}=y_{0}+h^{\alpha}\left(\frac{a_{1} x_{n-1} y_{n-1}}{1+b_{1} x_{n-1}}-\frac{a_{2} y_{n-1} z_{n-1}}{1+b_{2} y_{n-1}}-\mu_{1} y_{n-1}-\eta y_{n-1}^{2}+\sigma_{2} y_{n-1} \sqrt{h} \zeta_{2 n}\right)-\sum_{j=1}^{n} w_{j}^{\alpha}\left(y_{n-j}-y_{0}\right) \\
& z_{n}=z_{0}+h^{\alpha}\left(\frac{a_{2} y_{n-1} z_{n-1}}{1+b_{2} y_{n-1}}-\mu_{2} z_{n-1}+\sigma_{3} z_{n-1} \sqrt{h} \zeta_{3 n}\right)-\sum_{j=1}^{n} w_{j}^{\alpha}\left(z_{n-j}-z_{0}\right),
\end{aligned}
$$


where, $\sigma_{i}$ and $\zeta_{i}(n)$ represent real constants and a 3D Gaussian white noise processes, respectively, $i=1,2,3 . \zeta_{i}$ satisfy the follows:

$\left\langle\zeta_{j}(t)\right\rangle=0,(j=0,1,2,3)$ and $\left\langle\zeta_{i}\left(t_{1}\right) \zeta_{j}\left(t_{2}\right)\right\rangle=\delta_{i j} \delta\left(t_{1}-t_{j}\right)$,

$\delta_{i j}$ is Kronecker delta and $\delta\left(t_{1}-t_{j}\right)$ is the Dirac delta function.

\section{Numerical Simulations}

In this part, the numerical simulations of the mathematical model (3) will be examined, and the focus will be on the effect of harvesting parameters. The numerical results will be compared with the theorems formulated in the previous sections. The interactions between prey, middle predator and the top predator will be simulated by the following parameters: $a_{1}=2, a_{2}=0.2, b_{1}=$ $2, b_{2}=1, h=0.1, \mu_{1}=0.1, \mu_{2}=0.02$.

In order to show the effects of fractional derivative $\alpha$ on the dynamics of system (3) one can draw the bifurcation diagram considering fractional-order $\alpha$ as a bifurcation parameter. The supercritical Hopf bifurcation value centralizes at $\alpha^{*}=0.912047$ as indicated in Fig. 1 . When $\alpha<$ $\alpha^{*}$, the coexistence equilibrium point $E_{3}(0.8155,0.1111,2.88787)$ is locally asymptotically stable as shown in Fig. 1 and coincide with Fig. 2 when $\alpha=0.9$. For $\alpha>\alpha^{*}$, the system undergoes a limit cycle oscillations as exhibits in Fig. 1 and coincide with Fig. 2 when $\alpha=0.92$.

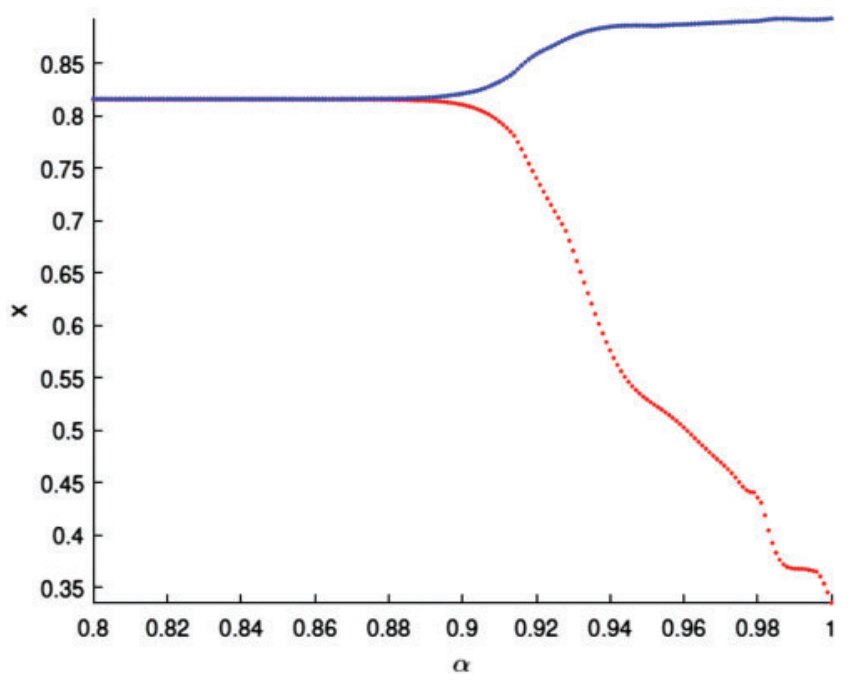

Figure 1: Bifurcation diagram of the fractional-order system (3) with respect to $\alpha$

For better understand the effect of the quadratic harvesting of the middle predator $\eta$ around the positive equilibrium point $E_{3}$, one can draw the bifurcation diagram with respect to $\eta$ as a bifurcation parameter. It can be seen that the supercritical Hopf bifurcation value localized at $\eta^{*}=0.229688$ as shown in Fig. 3. It can also be observed that when $\eta<0.229688$ the fractionalorder system (3) undergoes limit cycle behaviour as shown in Fig. 3 and coincide with Fig. 4 when $\eta=0.15$. For $\eta>0.229688$ the interior equilibrium point $E_{3}=(0.815539,0.111111,2.64157418)$ is locally asymptotically stable as indicated in Fig. 3 and coincide with Fig. 4 when $\eta=0.4$. It can 
also be observed that the conditions of local stability that are proven in Theorem 7 are verified because $\frac{a_{1} b_{1} y_{3}}{\left(1+b_{1} x_{3}\right)^{2}}=0.0642022<1$ and $\frac{a_{2} b_{2} z_{3}}{\left(1+b_{2} y_{3}\right)^{2}}=0.027935<\eta^{*}$.
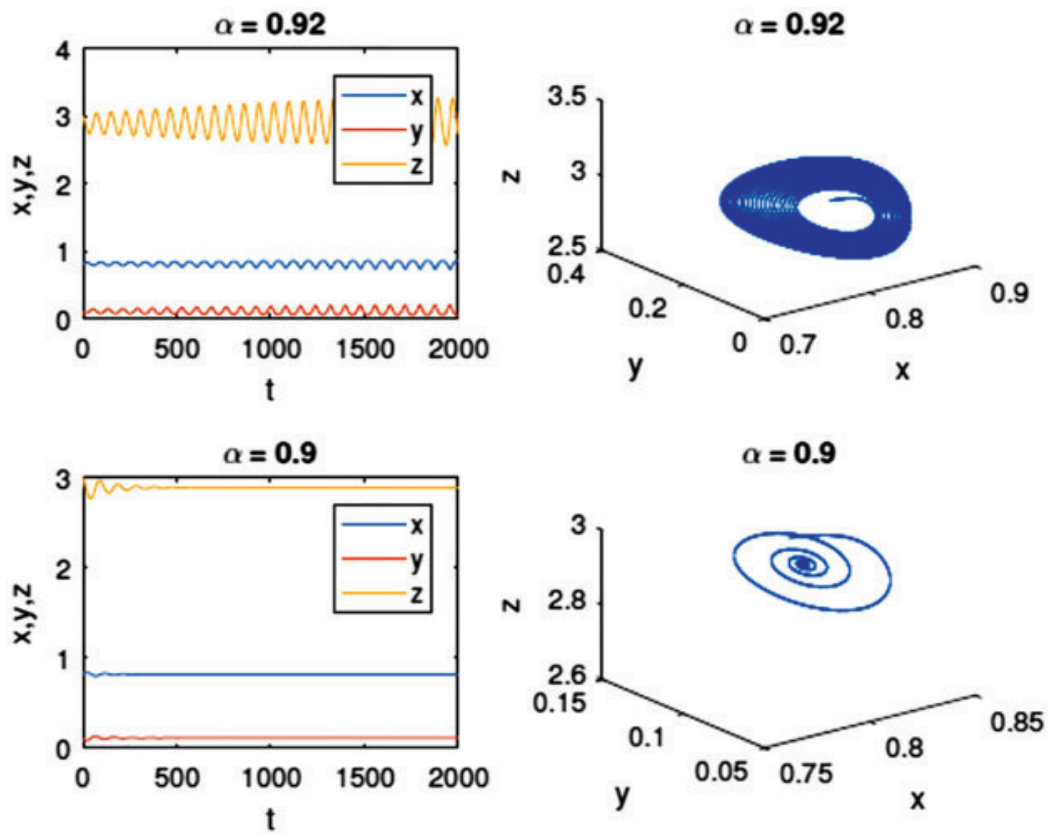

Figure 2: Time series and phase diagram of the equilibrium point $E_{3}$ of model (3) with different values of $\alpha$
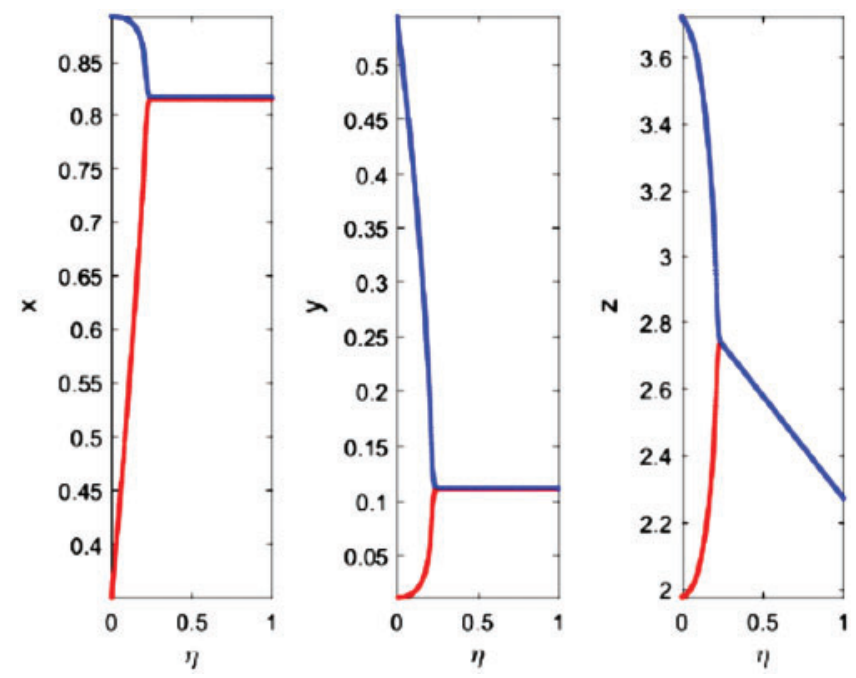

Figure 3: Bifurcation diagram of the fractional-order system (3) with respect to $\eta$

The effect of prey harvesting rate $h$ is shown in Fig. 5. From the bifurcation diagram with respect to $h$ as a bifurcation parameter, it can be seen that the supercritical Hopf bifurcation 
value localized at $h^{*}=0.3583$ as shown in Fig. 5. For $h<0.3583$ the fractional-order system (3) undergoes limit cycle behaviour as indicated in Fig. 6 when $h=0.2$. It can also be observed that when $h>0.3583$ the interior equilibrium point is locally asymptotically stable as shown in Fig. 5 and coincide with Fig. 6 when $h=0.4$.
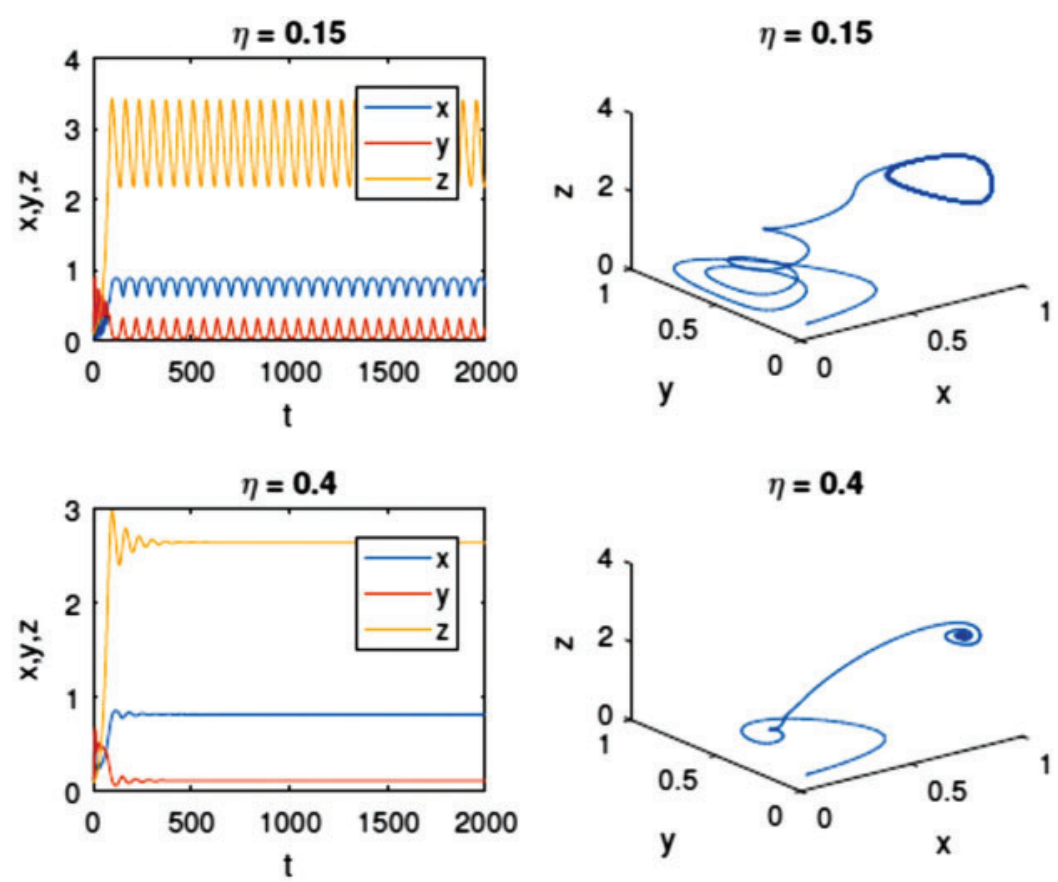

Figure 4: Time series and phase diagram of the equilibrium point $E_{3}$ of model (3) with different values of $\eta$

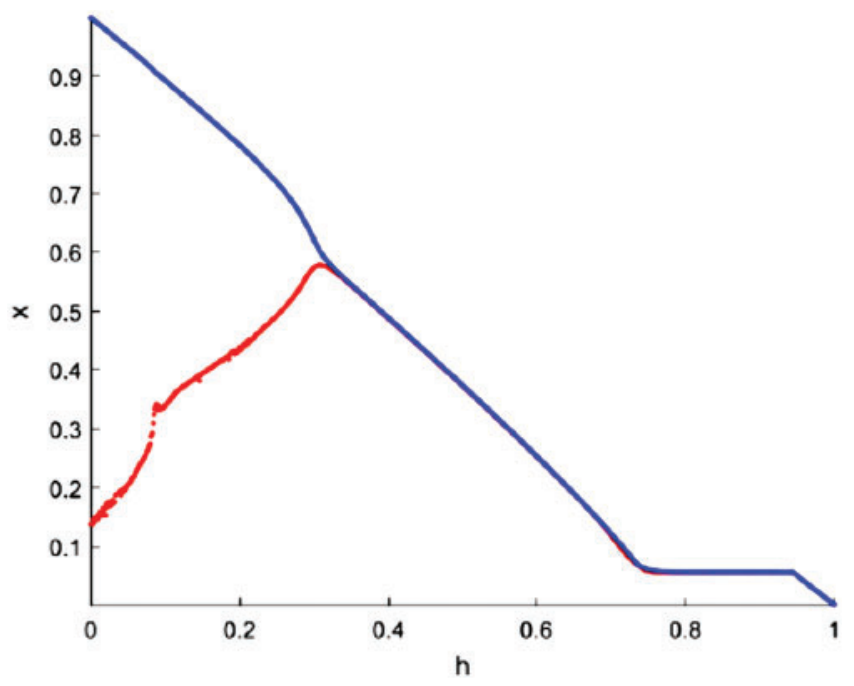

Figure 5: Bifurcation diagram of fractional-order system (3) with respect to $h$ 

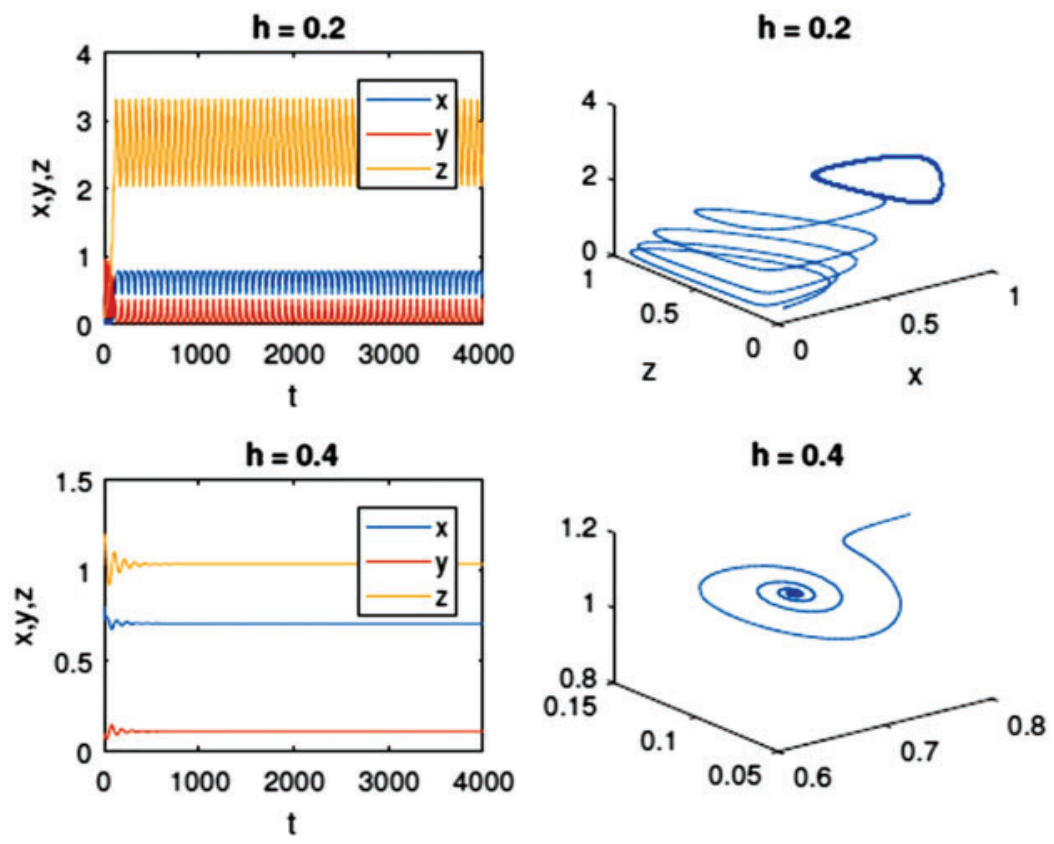

Figure 6: Time series and phase diagram of the equilibrium point $E_{3}$ of model (3) with $h=0.02$ and $h=0.4$

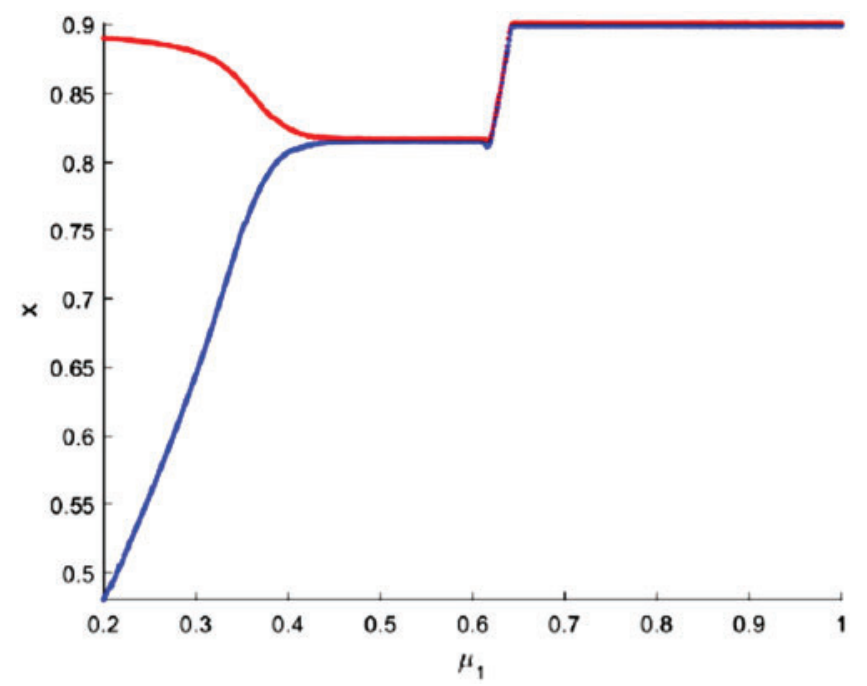

Figure 7: Bifurcation diagram of fractional-order system (3) with respect to $\mu_{1}$

If we increase the value of middle predator death rate $\mu_{1}$ and keeping all other parameters value fixes, it can be seen that a transcritical bifurcation occurs at $\mu_{1}=0.642857$ as shown in Fig. 7 and stated in Theorem 9. For $\mu_{1}<0.642857$ the interior equilibrium point $E_{3}=$ $(0.911269,0.111111,1.36443)$ is locally asymptotically stable as indicated in Fig. 7 and coincide with Fig. 8 when $\mu_{1}=0.4$. For $\mu_{1}>0.642857$ the free predator equilibrium point $E_{3}=(0.99,0,0)$ is locally asymptotically stable as shown in Fig. 7 and coincide with Fig. 8 when $\mu_{1}=0.7$. 

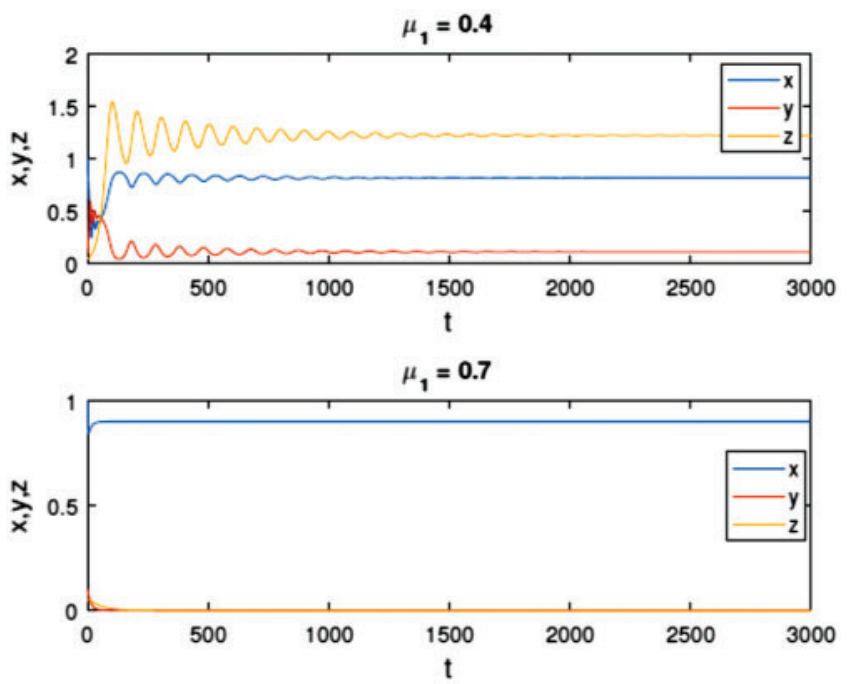

Figure 8: Time series of the food chain model (3) with $\mu_{1}=0.4$ and $\mu_{1}=0.7$

\section{Conclusion}

In this paper, a deterministic and stochastic fractional-order model of the tri-trophic food chain model incorporating harvesting has been proposed. It is shown that the proposed model has bounded and non-negative solution as desired in any population dynamics. By using stability analysis of fractional-order system, we have proved that if the basic reproduction number $R_{0}<1$, the predator free equilibrium point $E_{1}$ is globally asymptotically stable. The interaction between prey, the middle predator, and the top predator was investigated. The impact of harvesting on prey and middle predator was studied. Some sufficient conditions that ensure the local and global stability of equilibrium points have been obtained. We conclude that harvesting parameters can control the dynamics of the middle predator. A numerical approximation method is developed for the proposed stochastic fractional-order model.

Funding Statement: The authors gratefully acknowledge Qassim University, represented by the Deanship of Scientific Research, on the financial support under the number (cosao-bs-2019-2-2-I5469) during the academic year $1440 \mathrm{AH} / 2019 \mathrm{AD}$.

Conflicts of Interest: The authors declare that they have no conflicts of interest to report regarding the present study.

\section{References}

[1] A. Hastings and T. Powell, "Chaos in a three-species food chain," Ecology, vol. 72, no. 3, pp. 896-903, 1991.

[2] B. Buonomo, F. Giannino, S. Saussure and E. Venturino, "Effects of limited volatiles release by plants in tritrophic interactions," Mathematical Biosciences and Engineering, vol. 16, no. 5, pp. 3331-3344, 2019.

[3] P. Ghosh, P. Das and D. Mukherjee, "Chaos to order-Effect of random predation in a Holling type IV tri-trophic food chain system with closure terms," International Journal of Biomathematics, vol. 9, no. 5, pp. 1650073, 2016.

[4] K. Cheng, H. You and T. Yang, "Dynamics of three species food chain model with Holling type II functional response," arXiv preprint arXiv: 2004.12237v1, 2020. 
[5] B. Sahoo and S. Poria, "The chaos and control of a food chain model supplying additional food to top-predator," Chaos, Solitons \& Fractals, vol. 58, pp. 52-64, 2014.

[6] S. G. Mortoja, P. Panja, A. Paul, S. Bhattacharya and S. K. Mondal, "Is the intermediate predator a key regulator of a tri-trophic food chain model?: An illustration through a new functional response," Chaos, Solitons \& Fractals, vol. 132, no. 4, pp. 109613, 2020.

[7] N. Pal, S. Samanta and J. Chattopadhyay, "Revisited Hastings and Powell model with omnivory and predator switching," Chaos, Solitons \& Fractals, vol. 66, no. 12, pp. 58-73, 2014.

[8] N. Pal, S. Samanta and S. Rana, "The impact of constant immigration on a tri-trophic food chain model," International Journal of Applied and Computational Mathematics, vol. 3, no. 4, pp. 3615-3644, 2017.

[9] S. Raw, B. Tiwari and P. Mishra, "Analysis of a plankton-fish model with external toxicity and nonlinear harvesting," Ricerche di Matematica, vol. 69, no. 2, pp. 653-681, 2020.

[10] M. Haque, N. Ali and S. Chakravarty, "Study of a tri-trophic prey-dependent food chain model of interacting populations," Mathematical Biosciences, vol. 246, no. 1, pp. 55-71, 2013.

[11] B. Ghosh, D. Pal, T. Legoviác and T. Kar, "Harvesting induced stability and instability in a tri-trophic food chain," Mathematical Biosciences, vol. 304, no. 5, pp. 89-99, 2018.

[12] B. Nath, N. Kumari, V. Kumar and K. P. Das, "Refugia and Allee effect in prey species stabilize chaos in a tri-trophic food chain model," Differential Equations and Dynamical Systems, vol. 3, no. 12, pp. 591, 2019.

[13] A. Matouk, A. Elsadany, E. Ahmed and H. Agiza, "Dynamical behavior of fractional-order HastingsPowell food chain model and its discretization," Communications in Nonlinear Science and Numerical Simulation, vol. 27, no. 1-3, pp. 153-167, 2015.

[14] M. N. Huda, T. Trisilowati and A. Suryanto, "Dynamical analysis of fractional-order Hastings-Powell food chain model with alternative food," Journal of Experimental Life Science, vol. 7, no. 1, pp. 39-44, 2017.

[15] P. Panja, "Stability and dynamics of a fractional-order three-species predator-prey model," Theory in Biosciences, vol. 138, no. 2, pp. 251-259, 2019.

[16] L. Wang, H. Chang and Y. Li, "Dynamics analysis and chaotic control of a fractional-order threespecies food-chain system," Mathematics, vol. 8, no. 3, pp. 409, 2020.

[17] L. Addison, "Analysis of a predator-prey model: A deterministic and stochastic approach," J. Biom. Biostat., vol. 8, no. 4, pp. 359-368, 2017.

[18] R. Ali, M. S. Arif, M. Rafiq, M. Bibi and R. Fayyaz, "Numerical analysis of stochastic vector borne plant disease model," Computers, Materials \& Continua, vol. 63, no. 1, pp. 65-83, 2020.

[19] B. Ahmad, S. K. Ntouyas, A. Alsaedi and A. F. Albideewi, "A study of a coupled system of Hadamard fractional differential equations with nonlocal coupled initial-multipoint conditions," Advances in Difference Equations, vol. 2021, no. 1, pp. 1-16, 2021.

[20] S. Ahmad, R. Ullah and D. Baleanu, "Mathematical analysis of tuberculosis control model using nonsingular kernel type Caputo derivative," Advances in Difference Equations, vol. 2021, no. 1, pp. 1-18, 2021.

[21] R. Almeida, A. M. B. da Cruz, N. Martins and M. T. T. Monteiro, "An epidemiological MSEIR model described by the Caputo fractional derivative," International Journal of Dynamics and Control, vol. 7, no. 2, pp. 776-784, 2019.

[22] N. Sweilam, S. Al-Mekhlafi, A. Albalawi and D. Baleanu, "On the optimal control of coronavirus (2019-nCov) mathematical model; a numerical approach," Advances in Difference Equations, vol. 2020, no. 1, pp. 1-13, 2020.

[23] Q. Liu, D. Jiang, T. Hayat, A. Alsaedi and B. Ahmad, "Dynamical behavior of a higher order stochastically perturbed SIRI epidemic model with relapse and media coverage," Chaos, Solitons \& Fractals, vol. 139, no. Suppl. 3, pp. 110013, 2020.

[24] A. I. Aliyu, M. Inc, A. Yusuf and D. Baleanu, "A fractional model of vertical transmission and cure of vector-borne diseases pertaining to the Atangana-Baleanu fractional derivatives," Chaos, Solitons \& Fractals, vol. 116, pp. 268-277, 2018. 
[25] I. Tejado, E. Páerez and D. Valáerio, "Fractional derivatives for economic growth modelling of the group of twenty: Application to prediction," Mathematics, vol. 8, no. 1, pp. 50, 2020.

[26] K. Shah, F. Jarad and T. Abdeljawad, "On a nonlinear fractional order model of dengue fever disease under Caputo-Fabrizio derivative," Alexandria Engineering Journal, vol. 59, no. 4, pp. 2305-2313, 2020.

[27] B. Nath and K. P. Das, "Harvesting in tri-trophic food chain stabilises the chaotic dynamics-conclusion drawn from Hastings and Powell model," International Journal of Dynamical Systems and Differential Equations, vol. 10, no. 2, pp. 95-115, 2020.

[28] J. Cresson and A. Szafranáska, "Discrete and continuous fractional persistence problems-the positivity property and applications," Communications in Nonlinear Science and Numerical Simulation, vol. 44, no. 3, pp. 424-448, 2017.

[29] H. Li, L. Zhang, C. Hu, Y. Jiang and Z. Teng, "Dynamical analysis of a fractional-order predatorprey model incorporating a prey refuge," Journal of Applied Mathematics and Computing, vol. 54, no. 1-2, pp. 435-449, 2017.

[30] M. Sambath, P. Ramesh and K. Balachandran, "Asymptotic behavior of the fractional order three species prey-predator model," International Journal of Nonlinear Sciences and Numerical Simulation, vol. 19, no. 7-8, pp. 721-733, 2018.

[31] S. K. Choi, B. Kang and N. Koo, "Stability for Caputo fractional differential systems," Abstract and Applied Analysis, vol. 2014, no. 3-4, pp. 1-6, 2014.

[32] K. pada Das, "A mathematical study of a predator-prey dynamics with disease in predator," International Scholarly Research Network, vol. 807486, pp. 1-16, 2011.

[33] J. Huo, H. Zhao and L. Zhu, "The effect of vaccines on backward bifurcation in a fractional order HIV model," Nonlinear Analysis: Real World Applications, vol. 26, no. 3, pp. 289-305, 2015.

[34] M. S. Abdelouahab, N. E. Hamri and J. Wang, "Hopf bifurcation and chaos in fractional-order modified hybrid optical system," Nonlinear Dynamics, vol. 69, no. 1-2, pp. 275-284, 2012.

[35] L. Perko, Differential Equations and Dynamical Systems, vol. 7. New York, NY, USA: Springer, 2013.

[36] W. Wang, S. Cheng, Z. Guo and X. Yan, "A note on the continuity for Caputo fractional stochastic differential equations," Chaos: An Interdisciplinary Journal of Nonlinear Science, vol. 30, no. 7, pp. 73106, 2020.

[37] T. Doan, P. Huong, P. Kloeden and A. Vu, "Euler-maruyama scheme for Caputo stochastic fractional differential equations," Journal of Computational and Applied Mathematics, vol. 380, no. 4, pp. 112989, 2020.

[38] Z. Guo, J. Hu and W. Wang, "Caratheodory's approximation for a type of Caputo fractional stochastic differential equations," Advances in Difference Equations, vol. 2021, no. 1, pp. 68, 2021.

[39] D. T. Son, P. T. Huong, P. E. Kloeden and H. T. Tuan, "Asymptotic separation between solutions of Caputo fractional stochastic differential equations," Stochastic Analysis and Applications, vol. 36, no. 4, pp. 654-664, 2018.

[40] H. Aminikhah, A. H. R. Sheikhani, T. Houlari and H. Rezazadeh, "Numerical solution of the distributed-order fractional Bagley-Torvik equation," IEEE/CAA Journal of Automatica Sinica, vol. 6, no. 3, pp. 760-765, 2019.

[41] S. S. Ray and A. Patra, "Numerical solution of fractional stochastic neutron point kinetic equation for nuclear reactor dynamics," Annals of Nuclear Energy, vol. 54, no. 4, pp. 154-161, 2013.

[42] M. Hou, J. Yang, S. Shi and H. Liu, "Logical stochastic resonance in a nonlinear fractional-order system," European Physical Journal Plus, vol. 135, no. 9, pp. L453, 2020.

[43] G. Zou and B. Wang, "On the study of stochastic fractional-order differential equation systems," arXiv preprint arXiv: 1611.07618, 2016.

[44] I. Podlubny, Fractional Differential Equations. New York, NY, USA: Academic Press, 1999. 
[45] C. Huang, X. Yu, C. Wang, Z. Li and N. An, "A numerical method based on fully discrete direct discontinuous Galerkin method for the time fractional diffusion equation," Applied Mathematics and Computation, vol. 264, no. 1, pp. 483-492, 2015.

[46] C. Huang and M. Stynes, "Error analysis of a finite element method with GMMP temporal discretisation for a time-fractional diffusion equation," Computers \& Mathematics with Applications, vol. 79, no. 9, pp. 2784-2794, 2020. 